\title{
Video Article \\ V3 Stain-free Workflow for a Practical, Convenient, and Reliable Total Protein Loading Control in Western Blotting
}

\author{
Anton Posch ${ }^{1}$, Jonathan Kohn ${ }^{1}$, Kenneth $\mathrm{Oh}^{1}$, Matt Hammond ${ }^{1}$, Ning Liu ${ }^{1}$ \\ ${ }^{1}$ Bio-Rad Laboratories
}

Correspondence to: Ning Liu at Ning_liu@bio-rad.com

URL: https://www.jove.com/video/50948

DOI: doi:10.3791/50948

Keywords: Basic Protocol, Issue 82, Biotechnology, Pharmaceutical, Protein electrophoresis, Western blot, Stain-Free, loading control, total protein normalization, stain-free technology

Date Published: $12 / 30 / 2013$

Citation: Posch, A., Kohn, J., Oh, K., Hammond, M., Liu, N. V3 Stain-free Workflow for a Practical, Convenient, and Reliable Total Protein Loading Control in Western Blotting. J. Vis. Exp. (82), e50948, doi:10.3791/50948 (2013).

\section{Abstract}

The western blot is a very useful and widely adopted lab technique, but its execution is challenging. The workflow is often characterized as a "black box" because an experimentalist does not know if it has been performed successfully until the last of several steps. Moreover, the quality of western blot data is sometimes challenged due to a lack of effective quality control tools in place throughout the western blotting process. Here we describe the V3 western workflow, which applies stain-free technology to address the major concerns associated with the traditional western blot protocol. This workflow allows researchers: 1) to run a gel in about 20-30 min; 2) to visualize sample separation quality within 5 min after the gel run; 3) to transfer proteins in 3-10 min; 4) to verify transfer efficiency quantitatively; and most importantly 5) to validate changes in the level of the protein of interest using total protein loading control. This novel approach eliminates the need of stripping and reprobing the blot for housekeeping proteins such as $\beta$-actin, $\beta$-tubulin, GAPDH, etc. The V3 stain-free workflow makes the western blot process faster, transparent, more quantitative and reliable.

\section{Video Link}

The video component of this article can be found at https://www.jove.com/video/50948/

\section{Introduction}

Western blot is a very useful technique ${ }^{9}$, however, there are two major challenges with western blotting: long and labor intensive process and quality of data. A traditional protocol requires about 2 days. It involves many steps including sample preparation, gel casting, protein electrophoresis and transfer, membrane blocking followed by antibody incubation, imaging, and quite often stripping, reprobing, and finally data analysis. Throughout this process, there are no reliable and flexible tools for process control. As such, errors can be introduced at each step, and these errors have the potential to generate data artifacts; therefore, loading controls are essential in western blotting to identify and correct for the errors. The loading control is usually done by checking the protein level of a reference protein in each sample to see if it is equally presented. People often use housekeeping proteins, such as $\beta$-actin, $\beta$-tubulin, GAPDH, as loading control.

The quality of western blot data depends on reliable loading control. But there are two legitimate concerns when using housekeeping proteins for loading controls: 1) the antibody-based immunodetection of the housekeeping protein bands are often saturated and therefore one cannot distinguish the loading differences among the samples ${ }^{30} ; 2$ ) the housekeeping protein expression level may vary in the samples under certain experimental conditions, for example, siRNA treatment, cell death, cell differentiation, etc. ${ }^{11,28,3,6,10,21}$. Due to these concerns, scientific journals are now requiring that "for quantitative comparisons, appropriate reagents, controls and imaging methods with linear signal ranges should be used" (Nature guideline). Similarly, editors from the Journal of Clinical Investigation are asking for more reliable loading controls ${ }^{24}$. For these reasons, a housekeeping protein needs to be validated in order to be used as loading control. First, one has to make sure it is measured in the linear dynamic range of the immunodetection method ${ }^{14,29}$. Second, one has to make sure it is expressed consistently in all samples ${ }^{26,31,25,19,20}$.

An alternative solution to a reliable loading control is to use total protein measurement from the blot. Some researchers have stained the blots with total protein stains, such as Coomassie, Flamingo Pink, Sypro Ruby, Amido Black, Ponceau S, and stain-free technology, to measure the total protein signal in each lane as loading control ${ }^{16,20,13,27,1,4,12}$. The total protein loading control avoids the pitfalls associated with housekeeping proteins. First, it is a true reflection of the amount of protein loaded for each sample. Second, the total protein stain exhibits excellent linear dynamic range in the common loading range for western blot analysis (10-50 $\mu \mathrm{g}$ protein of a complex cell lysate) and accurately differentiates the loading difference among the samples ${ }^{12}$.

Stain-free technology is a novel total protein staining method where a unique compound is mixed in acrylamide gel solution and evenly distributed in the casted gel. After electrophoresis is completed, the gel is exposed to UV light for a minimum of 1 min so that the stain compound reacts with the tryptophan residues in the protein. The proteins become excitable under UV light to give a strong fluorescent signal that can be visualized and quantified in a stain-free enabled imager such as the ChemiDoc MP system. The stain-free compound itself, however, does not 
absorb UV light, resulting in low background of the gel image. The modification of the tryptophan residues is irreversible and proteins can be visualized not only in the gel but also on the blot at any time after protein transfer.

The stain-free technology is applied in the V3 Western Workflow (Figure 1) to address the major complaints about the traditional workflow, especially the concerns with using housekeeping proteins as loading controls. Using this workflow, one could: 1) run a gel in about 20-30 min, 2) check sample integrity and protein separation quality in 5 min after gel run; 3) transfer proteins in 3-10 min; 4) check the transfer efficiency quantitatively; and 5) most importantly, validate changes in the level of the protein of interest using total protein loading control.

\section{Protocol}

\section{Protein Sample Prep}

(A typical procedure to extract proteins from cell culture is described)

1. Place the HeLa cell culture dish in ice and wash the cells with ice-cold Tris-buffered Saline (TBS; $20 \mathrm{mM} \mathrm{Tris-HCl,} \mathrm{pH} 7.5,150 \mathrm{mM} \mathrm{NaCl}$ ).

2. Aspirate the TBS, then add $1 \mathrm{ml}$ per $100 \mathrm{~mm}$ dish ice-cold RIPA buffer $(50 \mathrm{mM}$ Tris- $\mathrm{HCl} \mathrm{pH} 8.0,150 \mathrm{mM} \mathrm{NaCl}, 1 \% \mathrm{NP}-40,0.5 \%$ sodium deoxycholate, $0.1 \%$ SDS) supplemented with phosphatase and protease inhibitors.

3. Scrape adherent cells off the dish using a cold plastic cell scraper; gently transfer the cell suspension into a precooled microcentrifuge tube.

4. Maintain constant agitation for $30 \mathrm{~min}$ at $4^{\circ} \mathrm{C}$ on a rotator.

5. Spin at $16,000 \times \mathrm{g}$ for $20 \mathrm{~min}$ in a $4^{\circ} \mathrm{C}$ precooled centrifuge.

6. Gently remove the tube from the centrifuge and place on ice. Transfer the supernatant to a fresh tube kept on ice, and discard the pellet.

7. Remove a small volume $(10-20 \mu \mathrm{l})$ of lysate to perform a protein assay. Determine the protein concentration for each sample using the RC DC assay kit.

8. If needed, aliquot the protein samples for long term storage at $-20^{\circ} \mathrm{C}$. Repeat freeze and thaw cycles cause protein degradation and should be avoided.

9. Take about $20 \mu \mathrm{g}$ of each sample, add an equal volume of $2 x$ Laemmli Sample Buffer (4\% SDS, $10 \%$ 2-mercaptoethanol, $20 \%$ glycerol, $0.004 \%$ bromophenol blue, $125 \mathrm{mM}$ Tris- $\mathrm{HCl}, \mathrm{pH} 6.8$ ).

10. Heat each cell lysate in sample buffer at $95^{\circ} \mathrm{C}$ for $5 \mathrm{~min}$.

11. Centrifuge at $16,000 \mathrm{xg}$ in a microcentrifuge for $1 \mathrm{~min}$.

\section{Gel Electrophoresis with Stain-free Gels ( $30 \mathrm{~min})$}

1. Take a Criterion TGX Any KD stain-free precast gel (a midi format gel), remove the comb and the tape from the bottom of the cassette.

2. Place the cassette in the Criterion cell and fill the integrated upper buffer chamber with $60 \mathrm{ml}$ running buffer (25 mM Tris, $190 \mathrm{mM}$ glycine, $0.1 \%$ SDS, pH 8.3). Rinse the wells with running buffer.

3. Fill each half of the lower buffer tank with $400 \mathrm{ml}$ running buffer to the marked fill line.

4. Load the protein samples and appropriate protein markers.

5. Place the lid on the tank, aligning the color-coded banana plugs with corresponding jacks on the lid.

6. Run the gel for $\sim 30 \mathrm{~min}$ at $200 \mathrm{~V}$ or $20 \mathrm{~min}$ at $300 \mathrm{~V}$.

\section{Stain-free Gel Imaging Using the Chemidoc MP System to Check Protein Separation Quality ( 5 min)}

1. Remove the gel cassette from the cell. Use the gel cassette opening tool in the Criterion Cell lid to crack open the cassette and release the gel.

2. Apply a couple of milliliters of water to the center of the UV sample tray of the ChemiDoc MP imager. Carefully lift the gel from the cassette and place it on the tray.

3. Launch Image Lab software and capture the stain-free gel image (Figure 1A) with the following settings:

Application: stain-free gel

Gel activation time: 1 min

Imaging area: criterion gel

Image exposure time: automatically optimized for the most intense bands

4. Remove the gel from the sample tray and proceed immediately to transfer step.

\section{Protein Transfer with the Trans-Blot Turbo System ( 10 min)}

1. Open a Trans-Blot Turbo Midi PVDF Transfer Pack; place the bottom stack (which includes the membrane) on the base of the transfer cassette.

2. Place the gel on top of the membrane, place the top stack on the gel, and roll out bubbles.

3. Place the lid on the cassette base and turn the dial to lock it.

4. Insert the cassette into the blotter bay.

5. Start the transfer by selecting a preset Turbo program and choosing the Criterion gel size (midi), and then press RUN. A typical run takes only 7 min.

6. When the transfer is over, disassemble the blotting sandwich and place both the blot and the gel in a container with deionized water. 


\section{Stain-free Gel and Blot Imaging Using the Chemidoc MP System to Check Protein Transfer Efficiency and Quality ( 5 min)}

1. Place the post-transfer gel on the sample tray of the ChemiDoc MP imager.

2. Launch Image Lab software and capture the stain-free image of the post-transfer gel (Figure 1B) with the following settings:

Application: stain-free gel

Gel activation time: none

Imaging area: criterion gel

Image exposure time: same to the exposure time for the pretransfer gel image

3. Remove the gel from the sample tray and then image the blot (Figure 1C) with the following settings. Keep the blot wet with a few drops of water or TBST when imaging.

Application: stain-free blot

Imaging area: criterion gel

Image exposure time: automatically optimized for the most intense bands

4. Remove the blotting membrane from the sample tray and place it in a container with TBST ( $0.1 \%$ Tween 20 in TBS).

\section{Antibody Incubation}

1. Block by placing the blot in a solution of $3 \%$ bovine serum albumin (BSA) in TBST at room temp for $1 \mathrm{hr}$.

2. Incubate the blot overnight at $4{ }^{\circ} \mathrm{C}$ in the solution containing mouse primary antibody raised against the first target protein and rabbit primary antibody raised against the second target protein.

3. Pour out the solution containing the primary antibody. Next, wash the blot by agitating in $20 \mathrm{ml}$ of TBST for $5 \mathrm{~min}$. Repeat $4 x$ for a total of 5 washes.

4. Incubate for $1 \mathrm{hr}$ at room temp in the secondary antibody solution containing a Dylight 650 conjugated Goat-anti-mouse antibody and a Dylight 549 conjugated Goat-anti-rabbit antibody.

5. Pour out the solution containing the primary antibody. Next, wash the blot by agitating in $20 \mathrm{ml}$ of TBST for $5 \mathrm{~min}$. Repeat $4 \mathrm{x}$ for a total of 5 washes.

\section{Imaging and Data Analysis by Image Lab Software- Total Protein Normalization ( 5 min)}

1. Acquire a multiplexing fluorescent image of the blot (Figure 1E) by opening a new multichannel protocol, configure three fluorescent channels and run the protocol.

Channel 1:

Application: blot Dylight 650

Imaging area: criterion gel

Image exposure time: automatically optimized for the most intense bands

Channel 2:

Application: blot Dylight 549

Imaging area: criterion gel

Image exposure time: automatically optimized for the most intense bands

Channel 3:

Application: stain-free blot

Imaging area: criterion gel Image exposure time: automatically optimized for the most intense bands

2. Click the Normalization icon from the Analysis Tool Box and click Yes to detect lanes and bands.

3. Select and use "Lanes and Bands tools" to make adjustments to the lanes and bands if needed.

4. Select stain-free image as the normalization channel.

5. Select MW Analysis Tools and assign the MW standard lanes by checking the boxes below them.

6. To view the normalized volumes, click the Analysis Table on the tool bar. All calculations will be performed automatically by the software, including the Normalization Factor and Normalized Volumes. The target protein band intensity values are now adjusted for variation in the protein load. This will allow accurate comparisons of target proteins among the samples.

\section{Representative Results}

1. Assessment of sample integrity, protein separation quality, and transfer efficiency with stain-free gel images.

Protein extract from HeLa cells were separated at $300 \mathrm{~V}$ for $20 \mathrm{~min}$ on a 18-well Criterion AnyKD TGX stain-free gel. The protein samples were loaded $3 x$ at four different amounts (Lanes 1-3, $40 \mu \mathrm{g}$; Lanes 4-6, $30 \mu \mathrm{g}$; Lanes 7-9, $20 \mu \mathrm{g}$; Lanes 10-12, 10 $\mu \mathrm{g}$ ). The gel was activated under UV light for $1 \mathrm{~min}$. Figure 2A shows the gel image acquired right after the protein separation. Protein sample integrity (e.g. degradation) and separation quality (e.g. protein precipitation) can be visually assessed with this gel image. Proteins were then transferred for 7 min to a nitrocellulose membrane using Trans-Blot Turbo. Figure 2B shows the stain-free image of the post-transfer gel. Both images were acquired with the same exposure time $(6.8 \mathrm{sec})$. Lane 3 and 12 were selected to measure the transfer efficiency. Using the volume tool in the Image Lab software, a rectangular box (blue) was drew to cover lane 3 and 12 on both gel images. The calculation based on the volume values from these boxes indicated that transfer efficiency of both lanes was 80\% (Figure 2C). In this experiment, the AnyKD TGX gel was selected to study small to medium size target proteins and it was not optimized for the transfer of large proteins. Optimizing the transfer efficiency would require the use of lower percentage gel (e.g. 4-20\%) and/or an adjustment of the transfer time to facilitate the transfer of large proteins. 2. Stain-free total protein 
loading control is a reliable alternative to housekeeping loading control in western blotting to quantify a small change in the level of protein of interest.

MCM-7 is a DNA licensing replication factor the level of which decreases by $20-50 \%$ in Lymphoblastoid cell lines (LCL) after irradiation treatment. In this experiment, lysates ( $30 \mu \mathrm{g}$ each) of four control and irradiation-treated Lymphoblastoid cell line (LCL) cultures were separated on a 12-well Criterion AnyKD TGX stain-free gel. The gel was activated for 1 min under UV light and transferred by Trans-Blot Turbo to a PVDF membrane for immunoblotting. The housekeeping protein GAPDH (green) was probed with a rabbit antibody (Cell Signaling Technology, USA, $1: 2,500$ ) and a Dylight 549 conjugated Goat-anti-rabbit antibody (Rockland, USA, 1:20,000). The protein of interest MCM-7 (red) was probed using a mouse antibody (Abcam, USA, 1:1,000) and a Dylight 649 conjugated Goat-anti-mouse antibody (Rockland, 1:10,000).

Figure 3A shows a multiplex fluorescent image of total proteins (blue), MCM-7 (red) and GAPDH (green) detected in four control and irradiation treated LCL samples. Figure 3B is a stain-free image of the same blot showing the total protein patterns in each sample ( $30 \mu \mathrm{g})$. Image lab software selected the sample lanes (blue boxes) to measure MCM-7, GAPDH, and total protein volume in each lane. The MCM-7 levels were normalized either against the stain-free total protein measurement or against GAPDH. The normalized MCM-7 protein levels were statistically analyzed and the average MCM-7 protein band volume and standard deviation $(n=4)$ are presented in the chart (Figure 3C). Both normalization methods revealed a small decrease (about $25 \%$ ) in MCM-7 protein levels after irradiation treatment. The data with the total protein normalization exhibited a smaller standard deviation than that with GAPDH as the loading control. 


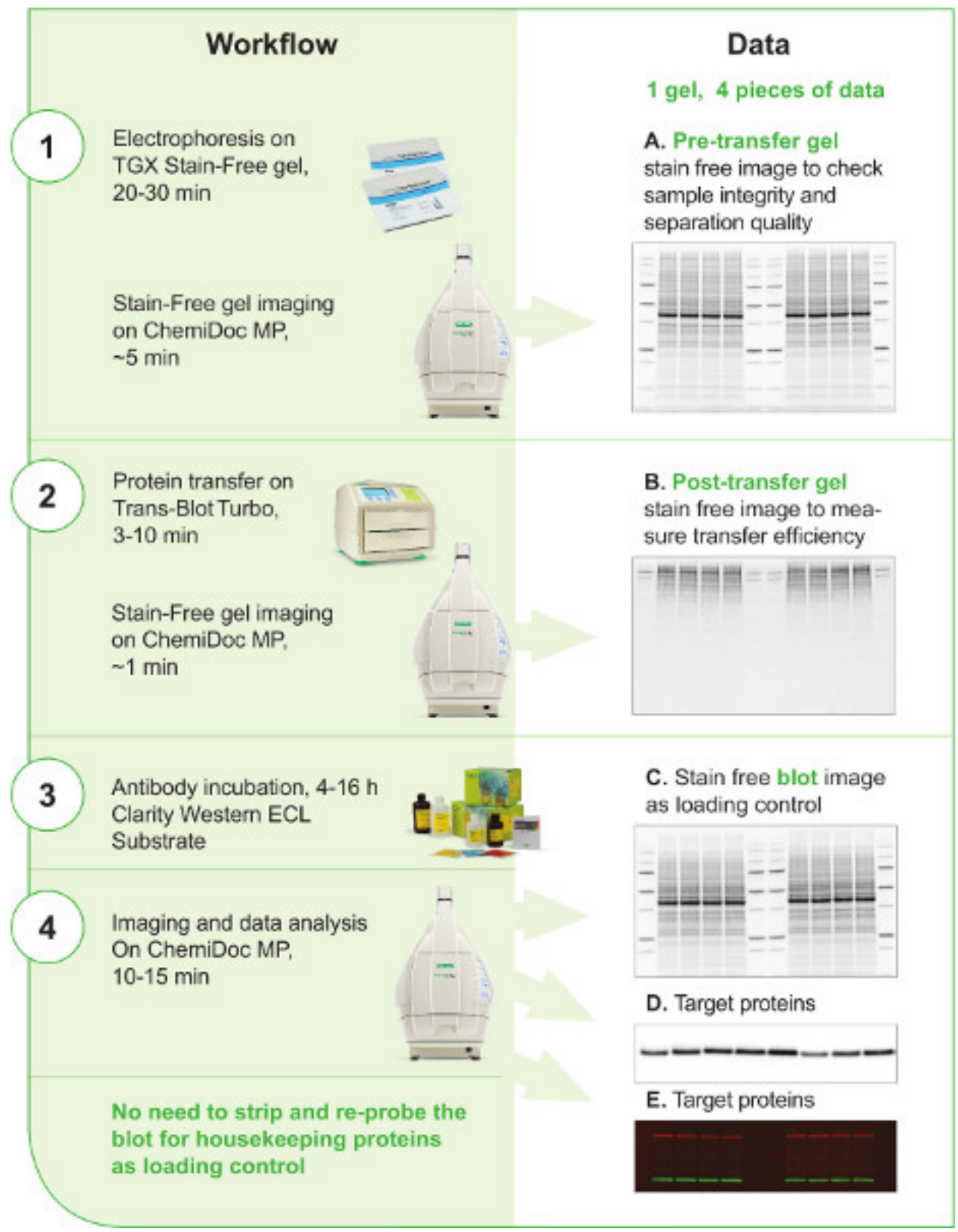

Figure 1. V3 Western Workflow. The V3 workflow is depicted in the left column in 4 steps. The major instruments and reagents used in the workflow are shown at each step. The estimated time for each step is also included. The right column shows that a minimum of 4 images can be generated in the V3 workflow. The use of each piece of data is described. The stain-free images of the pretransfer gel, post-transfer gel, and the blot (A, B, C) can not be generated easily with traditional western blotting techniques; These images and data provide important information and checkpoints along the procedure to improve the scientist's control and reproducibility of western blot workflow. The target protein signals can be captured either on a chemiluminescent blot image (D) if a HRP-conjugated secondary antibody was applied in detection or on a fluorescent blot image $(E)$ if multiplexing fluorescent western blotting was performed to detect more than one target protein simultaneously on the same blot. Click here to view larger image. 


\section{A. Pre-transfer gel stain-free image}

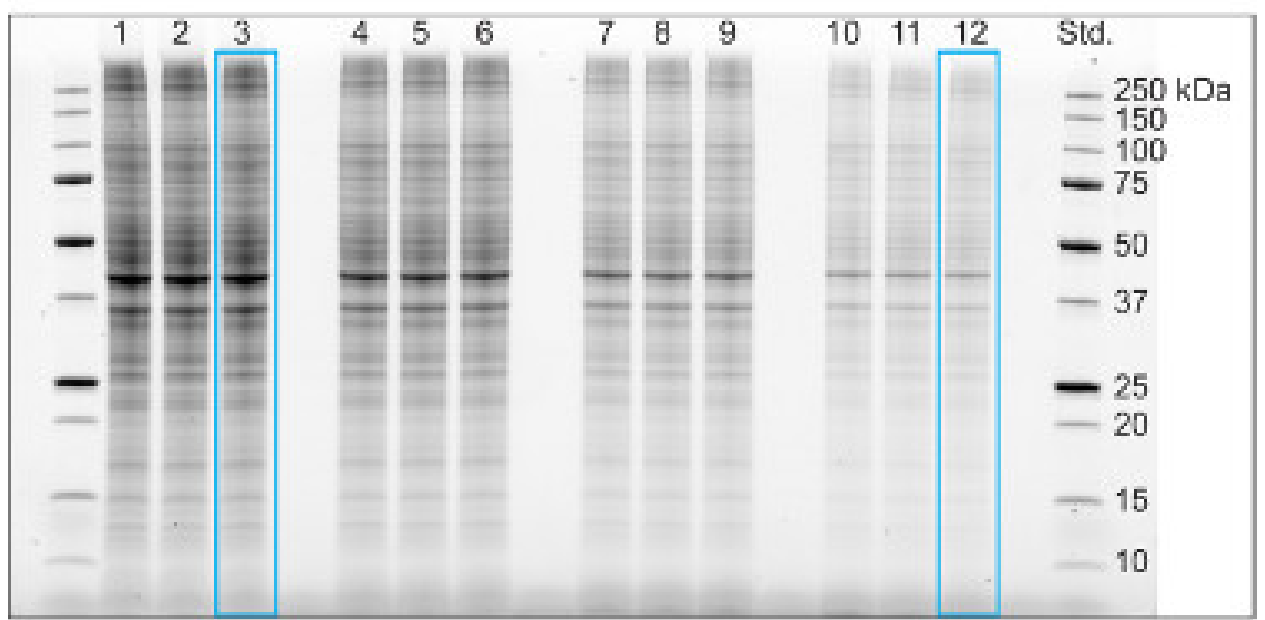

\section{B. Post-transfer gel stain-free image}

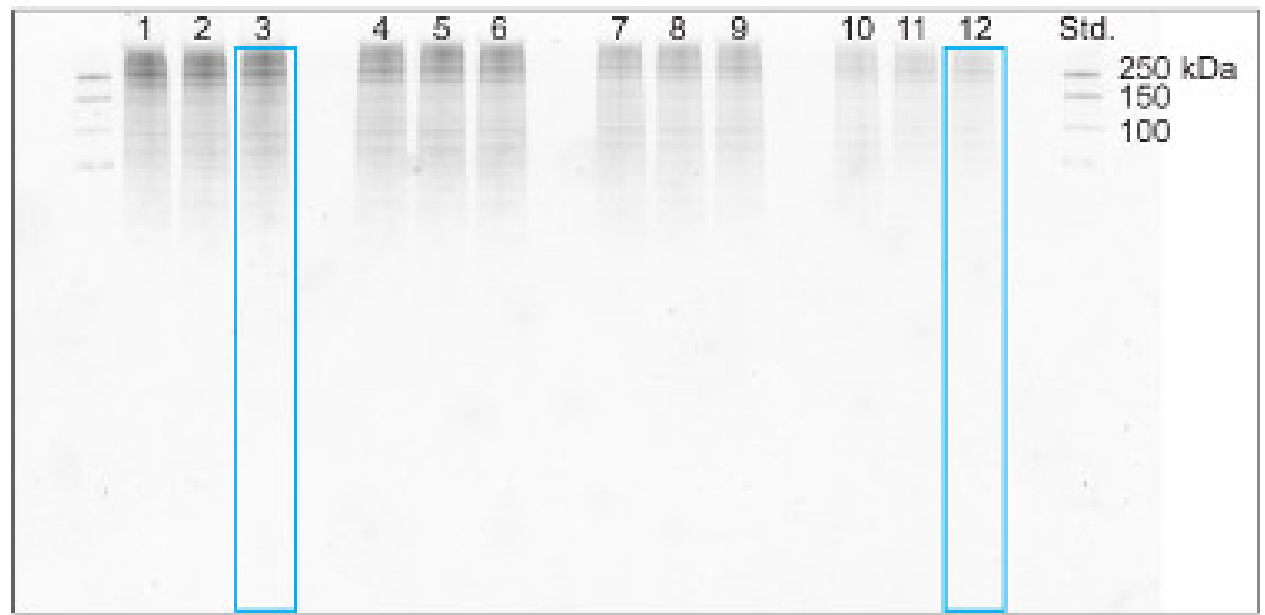

\section{Protein transfer efficiency measurement}

\begin{tabular}{|lcc|}
\hline & Lane 3 & Lane 12 \\
\hline Pre-Transfer lane volume & $397,248,155$ & $102,365,853$ \\
\hline Post-Transfer lane volume & $78,396,284$ & $20,069,698$ \\
\hline Protein transfer efficiency & $80.27 \%$ & $80.39 \%$ \\
\hline
\end{tabular}

Figure 2. Stain-free images of pretransfer and post-transfer gels in the V3 western workflow to assess sample integrity, separation quality, and transfer efficiency. (A) Pretransfer gel stain-free image. (B) Post-transfer gel stain-free image. (C) Protein transfer efficiency measurement. Click here to view larger image. 


\section{A. Fluorescent western blot image}

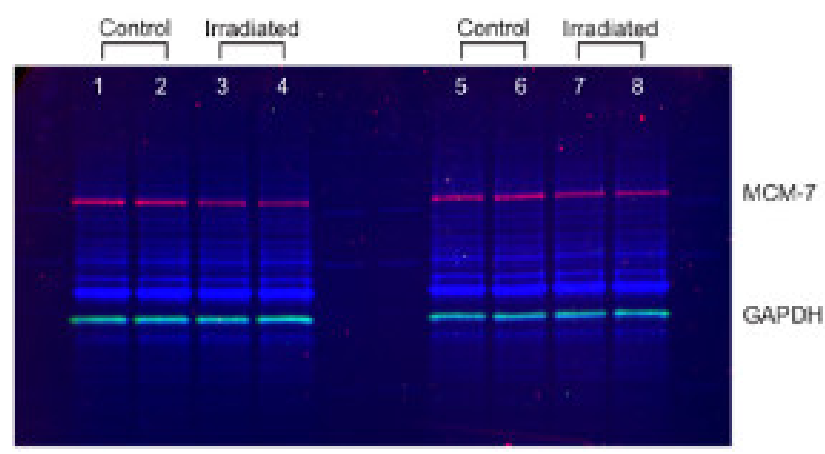

\section{B. Stain-free blot image}

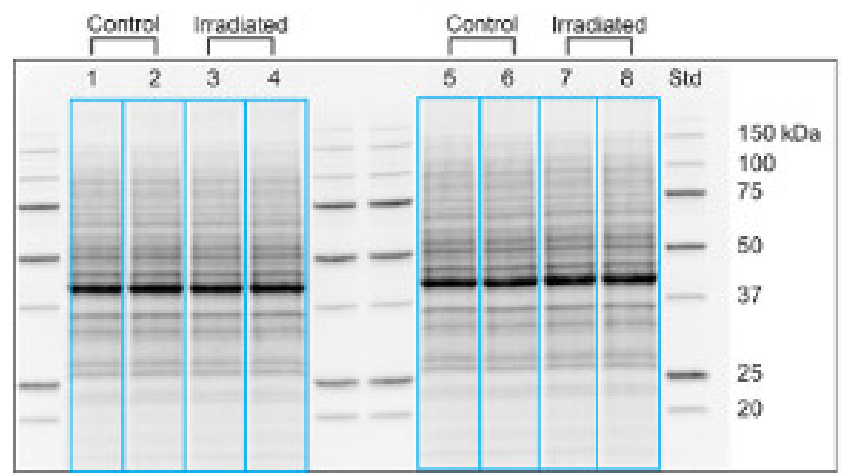

\section{Normalized MCM-7 protein levels}

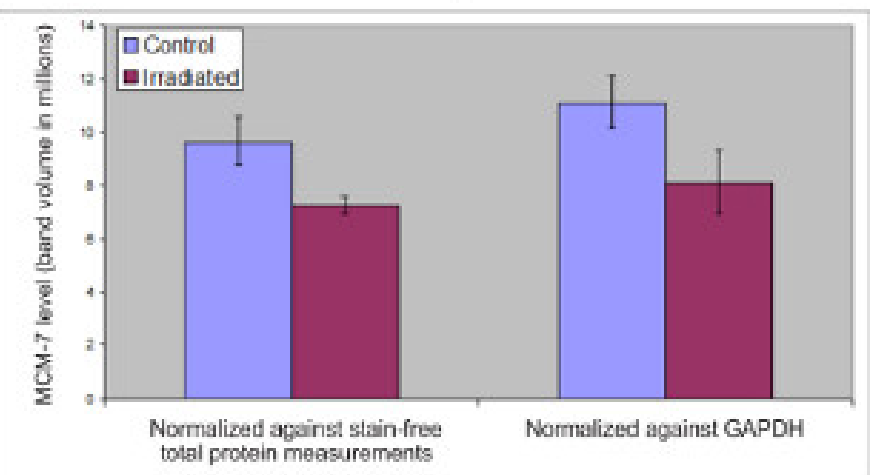

Figure 3. Comparing stain-free total protein measurement to GAPDH immunodetection as loading controls to normalize the level of protein of interest. (A) Fluorescent western blot image. (B) Stain-free blot image. (C) Normalized MCM-7 protein levels. Click here to view larger image.

\section{Discussion}

The V3 stain-free protocol described above is for multiplexing fluorescent western blotting. It can also be applied in western blotting using chemiluminescent detection. In the multiplex fluorescent western blot protocol, the stain-free blot images are acquired at two time points: 1) right after protein transfer; 2) at the multiplex fluorescent imaging step after antibody incubation. The first stain free image is used to calculate transfer efficiency and the second stain-free image is used as the loading control. When the chemiluminescent method is applied, it is not possible to take a multiplex image for the stain-free and target protein signals, because the chemiluminescent signal will show up in the stain-free channel too. In this case, we recommend to use the stain-free image taken right after the protein transfer step for loading control and normalization analysis.

The V3 western workflow provides the following unique benefits compared to the traditional western blot workflow that uses housekeeping proteins as loading controls: 
First, the V3 workflow provides a practical, convenient and more reliable loading control to validate the changes in the level of the protein of interest. The V3 protocol uses a total protein loading control to normalize the level of the protein of interest measured in each sample. It avoids two pitfalls of using the housekeeping proteins as loading controls: saturated immunodetection and inconsistent housekeeping protein expression levels among the samples under certain experimental conditions. Stripping and reprobing steps with antibodies directed against housekeeping are no longer necessary. Using stain-free technology, there is no need to stain and de-stain a blot with stains such as Coomassie or Sypro Ruby for total protein measurement. It takes just a few seconds to acquire a blot image and about 5 min to do total protein normalization using Image Lab software.

Second, the V3 workflow allows scientists to take better control of the western procedure because it makes the procedure more transparent and introduces several checkpoints for quality control. With the help of stain-free technology, researchers can visualize their protein samples on both the gel and the blot. Scientists can assess protein sample integrity (degraded or not), separation quality (precipitated or not), transfer efficiency and transfer quality (even transfer or not). These checkpoints help researches terminate the experiment when seeing big flaws in the process and avoid wasting time on poor samples and blots. This technology also helps scientists assess whether there is a significant amount of protein loss after membrane stripping and whether the blot is suitable for reprobing a different target ${ }^{4}$.

Here are a few tips to ensure good experience and quality data using V3 western workflow.

1. Image the gel immediately after gel run. Do not soak the gel in any buffer before the first imaging in the procedure as it may wash away the stain compound.

2. Use the same imaging area to acquire all images in the protocol. This will allow the software to overlay images for data analysis such as total protein normalization.

3. Keep the exposure time consistent when imaging the pretransfer and post-transfer gels. This will allow the software to quantitatively measure the transfer efficiency.

4. Keep the membrane wet with a few drops of water or TBST when acquiring the blot image. This will avoid possible dirty background problems for target protein detection.

5. Use low-fluorescent PVDF membrane for multiplexing fluorescent western blotting.

It is important to note that the stain-free molecule after UV-activation is irreversibly bound to tryptophan residues. This irreversible modification may potentially affect antigen recognition when using monoclonal antibodies if the epitope contains tryptophan. Polyclonal antibodies are unlikely to be affected because they recognize multiple epitopes on the antigen. Unbound stain-free molecules are easily washed off the gel and the membrane and therefore will not interfere with antibody-antigen interactions.

In conclusion, the V3 western workflow makes the western blot process faster, more transparent, quantitative and more reliable. Researchers now can easily apply total protein loading control in a western blot experiment to make their data more trustworthy. The V3 stain-free workflow has been adopted by a number of labs, and their publications have demonstrated that journals accept stain-free data as loading control in western blot $22,17,7,8,5,23,18,15$

\section{Disclosures}

The authors, Anton Posch, Jonathan Kohn, Kenneth Oh, Matt Hammond, and Ning Liu are employees of Bio-Rad Laboratories, Inc.

\section{Acknowledgements}

The authors thank Dr. Wolf-Dieter Stalz, Dr. Arnaud Remy, Dr. Anton Posch, Dr. Patricia Piatti, Tom Davies, Kris Simonyi, and Jeff Durban for their critical review and editing of this manuscript. The authors also thank Allison Schwartz for technical support.

\section{References}

1. Aldridge, G.M., Podrebarac, D.M., Greenough, W.T., \& Weiler, I.J. The use of total protein stains as loading controls: an alternative to highabundance single-protein controls in semi-quantitative immunoblotting. J. Neurosci. Methods. 172(2), $250-254$ (2008).

2. Bauer, D.E., Haroutunian, V., McCullumsmith, R.E., \& Meador-Woodruff, J.H. Expression of four housekeeping proteins in elderly patients with schizophrenia. J. Neural. Transm. 116(4), 487-491 (2009).

3. Castaño, Z. \& Kypta, R.M. Housekeeping Proteins: Limitations as References During Neuronal Differentiation. Open Neurosci. J. 2, 36-40 (2008).

4. Colella, A.D., Chegenii, N., Tea, M.N., Gibbins, I.L., Williams, K.A., \& Chataway, T.K. Comparison of Stain-Free gels with traditional immunoblot loading control methodology. Anal. Biochem. 430(2), 108-110 (2012).

5. Cully, T.R., Edwards, J.N., Friedrich, O., Stephenson, D.G., Murphy, R.M., \& Launikonis B.S. Changes in plasma membrane Ca-ATPase and stromal interacting molecule 1 expression levels for $\mathrm{Ca}^{2+}$ signaling in dystrophic mdx mouse muscle. Am. J. Physiol. Cell Physiol. 303(5), C567-C576 (2012).

6. Dittmer A and Dittmer, J., Beta-actin is not a reliable loading control in Western blot analysis. Electrophoresis. 27(14), 2844-2845 (2006).

7. Dutka, T.L., Lamboley, C.R., McKenna, M.J., Murphy, R.M., \& Lamb, G.D. Effects of carnosine on contractile apparatus Ca ${ }^{2+}$ sensitivity and sarcoplasmic reticulum $\mathrm{Ca}^{2+}$ release in human skeletal muscle fibers. J. Appl. Physiol. 112(5), 728-736 (2012).

8. Elliott, S., Busse, L., Swift, S., McCaffery, I., Rossi, J., Kassner, P., \& Begley, C.G. Lack of expression and function of erythropoietin receptors in the kidney. Nephrol. Dial. Transplant. 27(7), 2733-2745 (2012).

9. Eslami, A., and Lujan, J., Western Blotting: Sample Preparation to Detection. J. Vis. Exp. 44, e2359, doi:10.3791/2359 (2010)

10. Ferguson, R.E., Carroll, H.P., Harris, A., Maher, E.R., Selby, P.J., \& Banks, R.E. Housekeeping proteins: a preliminary study illustrating some limitations as useful references in protein expression studies. Proteomics. 5(2), 566-571 (2005). 
11. Greer, S., Honeywell, R., Geletu, M., Arulanandam, R., \& Raptis, L. Housekeeping genes; expression levels may change with density of cultured cells. J. Immunol. Methods. 355(1-2), 76-79 (2010).

12. Gürtler, A., Kunz, A., Gomolka, M., Hornhardt, S., Friedl, A.A., McDonald, K., Kohn, J.E., \& Posch, A, Stain-Free Technology as Normalization Tool in Western Blot Analysis. Anal. Biochem. 433(2), 105-111 (2013).

13. Hagiwara, M., Kobayashi, K., Tadokoro, T., \& Yamamoto, Y. Application of SYPRO Ruby- and Flamingo-stained polyacrylamide gels to Western blot analysis. Anal. Biochem. 397(2), 262-264 (2010).

14. Heidebrecht, F., Heidebrecht, A., Schulz, I., Behrens, S.E., \& Bader, A. Improved semiquantitative Western blot technique with increased quantification range. J. Immunol. Methods. 345(1-2), 40-48 (2009).

15. Jensen, R.B., Ozes, A., Kim, T., \& Estep, A. Kowalczykowski SC BRCA2 is epistatic to the RAD51 paralogs in response to DNA damage. DNA Repair. 2013 Feb 2. doi:pii: S1568-7864(13)00002-5. 10.1016/j.dnarep.2012.12.007. Epub ahead of print (2013).

16. Lanoix, D., St-Pierre, J., Lacasse, A.A., Viau, M., Lafond, J., \& Vaillancourt, C. Stability of reference proteins in human placenta: general protein stains are the benchmark. Placenta. 33(3), 151-156 (2012).

17. Larkins, N.T., Murphy, R.M., \& Lamb, G.D. Influences of temperature, oxidative stress, and phosphorylation on binding of heat shock proteins in skeletal muscle fibers. Am. J. Physiol. Cell Physiol. 3(6), C654-C565 (2012).

18. Laurie, K.J., Dave, A., Straga, T., Souzeau, E., Chataway, T., Sykes, M.J., Casey, T., Teo, T., Pater, J., Craig, J.E., Sharma, S., \& Burdon, K.P. Identification of a Novel Oligomerization Disrupting Mutation in CRYAA Associated with Congenital Cataract in a South Australian Family. Hum. Mutat. 2012 Dec 15. doi: 10.1002/humu.22260. Epub ahead of print (2012).

19. Li, X., Bai, H., Wang, X., Li, L., Cao, Y., Wei, J., Liu, Y., Liu, L., Gong, X., Wu, L., Liu, S., \& Liu, G. Identification and validation of rice reference proteins for western blotting. J. Exp. Bot. 62(14), 4763-4772 (2011).

20. Liu, N.K. \& Xu, X.M. Beta-tubulin is a more suitable internal control than beta-actin in western blot analysis of spinal cord tissues after traumatic injury. J. Neurotrauma. 23(12), 1794-1801 (2006).

21. Lowe, D.A., Degens, H., Chen, K.D., \& Alway, S.E. Glyceraldehyde-3-phosphate dehydrogenase varies with age in glycolytic muscles of rats. J. Gerontol. A Biol. Sci. Med. Sci. 55(3), B160-B164 (2000).

22. Mollica, J.P., Dutka, T.L., Merry, T.L., Lamboley, C.R., McConell, G.K., McKenna, M.J., Murphy, R.M., \& Lamb, G.D. S-glutathionylation of troponin I (fast) increases contractile apparatus Ca2+ sensitivity in fast-twitch muscle fibres of rats and humans. J. Physiol. 590(Pt 6), 1443-1463 (2012).

23. Murphy, R.M., Dutka, T.L., Horvath, D., Bell, J.R., Delbridge, L.M., \& Lamb, G.D. Ca2+-dependent Proteolysis of Junctophilin 1 and Junctophilin 2 in Skeletal and Cardiac Muscle. J. Physiol. 591(Pt 3), 719-729 (2012).

24. Neill, U.S., All Data are not created Equal. J. Clin. Invest. 119, 224 (2009).

25. Pérez-Pérez, R., López, J.A., García-Santos, E., Camafeita, E., Gómez-Serrano, M., Ortega-Delgado, F.J., Ricart, W., Fernández-Real, J.M., \& Peral, B. Uncovering suitable reference proteins for expression studies in human adipose tissue with relevance to obesity. PLoS One. 7(1), e30326 (2012).

26. Rocha-Martins, M., Njaine, B., \& Silveira, M.S. Avoiding Pitfalls of Internal Controls: Validation of Reference Genes for Analysis by qRT-PCR and Western Blot throughout Rat Retinal Development. PLoS One. 7(8), e43028 (2012).

27. Romero-Calvo, I., Ocón, B., Martínez-Moya, P., Suárez, M.D., Zarzuelo, A., Martínez-Augustin, O., \& de Medina, F.S. Reversible Ponceau staining as a loading control alternative to actin in Western blots. Anal. Biochem. 401(2), 318-320 (2010).

28. Said, H.M., Polat, B., Hagemann, C., Anacker, J., Flentje, M., \& Vordermark, D. Absence of GAPDH regulation in tumor-cells of different origin under hypoxic conditions in-vitro. BMC Res. Notes. 13(2), 8 (2009).

29. Suzuki, O., Koura, M., Noguchi, Y., Uchio-Yamada, K., \& Matsuda, J. Use of sample mixtures for standard curve creation in quantitative western blots. Exp. Anim. 60(2), 193-196 (2011).

30. Welinder, C. \& Ekblad, L. Coomassie staining as loading control in Western blot analysis. J. Proteome Res. 10(3), 1416-1419 (2011).

31. You, J., Hodge, C., Wen, L., McAvoy, J.W., Madigan, M.C., \& Sutton, G. Using soybean trypsin inhibitor as an external loading control for Western blot analysis of tear proteins: application to corneal disease. Exp. Eye Res. 99, 55-62 (2012). 